\title{
Influence of Optimism on Appraised Stress, Depression, and Anxiety Among College Students*
}

\author{
Chris A. Eisenbarth \\ Weber State University, Ogden, USA
}

\begin{abstract}
This study examined the direct and interactive effects of appraised stress and dispositional optimism in predicting symptoms of depression and anxiety among college students. Consistent with a stress-buffering hypothesis, the results suggest that students' appraised stress, depressive symptoms, and anxiety are moderated by optimism. The utility of this research for reducing symptoms of depression and anxiety among students rests on the modifiability of appraised stress by the promotion of an adaptive, future-oriented perspective (i.e., optimism).
\end{abstract}

Keywords: dispositional optimism, stress, depression, college students

\section{Introduction}

Research suggests that many college students are stressed by the demands of school (Robotham \& Julian, 2006) and experiencing symptoms of depression and anxiety (Kassel, Bornovalova, \& Metha, 2006; McCarthy, Fouladi, Juncker, \& Matheny, 2006). According to the ACHA-NCHA (American College Health Association-National College Health Assessment) (2010), stress is the leading impediment to students' academic performance and approximately one third of students report feeling stressed and overwhelmed by school. Furthermore, symptoms of depression and anxiety are generally more common among students than in the general population (Stewart-Brown, Patterson, Peterson, Doll, Balding, \& Regis, 2000). Given such findings, it is not surprising that college student stress, depression, and anxiety are matters of growing concern for researchers and health professionals.

A particularly relevant individual difference variable that may lessen appraised stress and influence depression and anxiety is dispositional optimism (i.e., positive outcome expectancies). Optimism is conceptualized as a stable, trait-like personality characteristic comprised of a general, positive attitude about the future and a tendency to anticipate favorable outcomes (Scheier, Carver, \& Bridges, 1994). The supposition is that someone with high dispositional optimism would be less likely to experience appraised stress, depression, and anxiety than someone with low levels of dispositional optimism. Some empirical support exists for these propositions as optimism has been linked to various measures of perceived stress and psychological adjustment (e.g., Chang, 1998; Chang \& Sanna, 2003; Huan, Yeo, Ang, \& Chong, 2006).

Despite clear conceptual linkages among appraised stress, optimism, depression, and anxiety there has

\footnotetext{
* This paper was presented in part at the Research Consortium Conference of the American Alliance of Health, Physical Education, Recreation, and Dance National Convention and Exposition, San Diego, March 31, 2011.

Chris A. Eisenbarth, Assistant Professor, Department of Health Promotion and Human Performance (HPHP), Weber State University.
} 
been surprisingly little empirical research with college students (e.g., Baldwin, Chamblis, \& Towler, 2003; Brissette, Scheier, \& Carver, 2002; Chang, 1998; Lai, 2009), and the dynamic interplay among these variables is in need of further study. To address this need, the purpose of the present study was to examine both the direct and interactive (or moderating) roles of appraised stress and optimism in predicting symptoms of depression and anxiety among college students.

\section{Method}

Participants $(n=353)$ consisted of male $(n=172)$ and female $(n=181)$ college students enrolled in large, baccalaureate core classes at a large university in the Northwest United States. Ninety percent of the sample was 18 to 20 years old, with less than three percent being older than age 25 . Approximately $80 \%$ of the sample identified themselves as White, European American, 9\% as Asian, with no other ethnic group exceeding three percent of the total sample. Prior to data collection, the project received institutional review board approval for the protection of human participants in research.

A cross-sectional, self-report survey format was used to collect data. Participants voluntarily completed a questionnaire that assessed demographic background information, appraised stress, dispositional optimism, depression, and anxiety. The perceived stress scale (Cohen, Kamarch, \& Mermelstein, 1983) was used to evaluate the nonspecific appraised stress of participants. Dispositional optimism was assessed with the revised life orientation test (Scheier et al., 1994). P. F. Lovibond and S. H. Lovibond's (1995) depression scale was used to measure symptoms of low positive effect and a sense of helplessness. Similarly, P. F. Lovibond and S. H. Lovibond's (1995) anxiety scale was used to assess symptoms of autonomic arousal (e.g., elevated heart rate) and fearfulness. Alpha coefficients for the aforementioned scales ranged from 0.74 to 0.87 in the present study and suggested that a high degree of internal consistency existed. Zero-order correlations were also computed among the study variables and ranged from $|0.47|$ to $|0.62|$ suggesting that the scales tapped relatively independent constructs (see Table 1).

Table 1

Variable Correlations, Means, SDs (Standard Deviations), and Alpha Coefficients

\begin{tabular}{llllll}
\hline & Scale & 1 & 2 & 3 & 4 \\
\hline 1 & Appraised stress & -- & & & \\
3 & Optimism & -0.47 & -- & & \\
4 & Depression & 0.62 & -0.48 & -- & -- \\
& Anxiety & 0.51 & -0.33 & 0.63 & 1.84 \\
& Mean & 2.64 & 2.13 & 2.01 & 0.68 \\
& $S D$ & 0.59 & 0.38 & 0.70 & 0.81 \\
\hline
\end{tabular}

Notes. All zero-order correlations are significant, $p<0.01$; Items 1-4 were assessed using 5-point Likert-type scales ranging from 1 ("Never") to 5 ("Very often").

\section{Results}

Hierarchical, multiple-regression analyses were conducted to evaluate the relative contributions of appraised stress and optimism in predicting symptoms of depression and anxiety. To account for potential differences among male and female participants on the variables of study, the sex variable was entered in 
the first step of the regression formulas. To examine the moderating (or stress-buffering) effect of optimism, an interaction term was created by multiplying perceived stress by optimism. To yield correct standardized solutions, all variables were centered before analysis. Results of these analyses are presented in Table 2.

Table 2

Summary of Hierarchical Regression Analysis for Variables Predicting Depression and Anxiety

\begin{tabular}{|c|c|c|c|c|}
\hline Variable & $R$ & $\Delta R^{2}$ & $d f$ & $F$ \\
\hline \multicolumn{5}{|l|}{ Depressive symptoms } \\
\hline Sex & 0.03 & 0.00 & 1,351 & $0.30^{*}$ \\
\hline Perceived stress & 0.62 & 0.39 & 1,350 & $111.69^{*}$ \\
\hline Optimism & 0.66 & 0.04 & 1,349 & $88.69^{*}$ \\
\hline Perceived stress $\times$ optimism & 0.69 & 0.04 & 1,348 & $78.10^{*}$ \\
\hline \multicolumn{5}{|l|}{ Anxiety symptoms } \\
\hline Sex & 0.18 & 0.03 & 1,351 & $11.22 *$ \\
\hline Perceived stress & 0.52 & 0.24 & 1,350 & $64.72^{*}$ \\
\hline Optimism & 0.53 & 0.01 & 1,349 & $88.69^{*}$ \\
\hline Perceived stress $\times$ optimism & 0.56 & 0.03 & 1,348 & $78.10^{*}$ \\
\hline
\end{tabular}
Note. $p<0.01$.

With depressive symptoms as the dependent variable, the sex variable was entered in the first step of the regression formula and was not significant. In the second step, perceived stress was found to account for a significant $39 \%$ of variance in depressive symptoms, $p<0.01$. Optimism was found to account for a significant four percent of additional variance in step three, $p<0.01$. The perceived stress $\times$ optimism interaction was found to account for another significant four percent of variance in step four, $p<0.01$. The full regression model including sex, perceived stress, dispositional optimism, and the interaction term accounted for $47 \%$ of the variance in depressive symptoms, $F_{(4,348)}=78.10, p<0.001$. Significant effects emerged for appraised stress $(\beta=0.52, p<0.01)$, optimism $(\beta=-0.26, p<0.01)$, and the interaction of optimism with appraised stress $(\beta=-0.20, p<0.01)$.

With anxiety as the dependent variable, sex accounted for a significant $3 \%$ of the variance in step one, $p$ $<0.01$. In the second step, perceived stress contributed a significant $24 \%$ of variance, $p<0.01$. In step 3 , the optimism variable added one percent of variance in anxiety symptoms, $p<0.02$. In the final step, the perceived stress $\mathrm{x}$ optimism interaction was found to account for a significant three percent of additional variance in students' self-reported anxiety, $p<0.01$. The full regression model including sex, perceived stress, dispositional optimism, and the interaction term accounted for $31 \%$ of the variance in anxiety symptoms, $F_{(4,348)}=39.05, p<0.001$. Significant effects emerged for $\operatorname{sex}(\beta=0.10, p<0.03)$, appraised stress $(\beta=0.43, p<0.01)$, optimism $(\beta=-0.14, p<0.01)$, and the interaction of optimism with appraised stress $(\beta=-0.17, p<0.01)$.

To determine the form of the interactions, regression lines were plotted to depict the regression of optimism on the outcome measures as a function of low (1 standard deviation below the mean) and high (1 SD above the mean) appraised stress (Aiken \& West, 1991). The form of the interactions suggested that decreases in optimism exacerbate depression and anxiety in instances of high appraised stress (see Figures 1 and 2). 


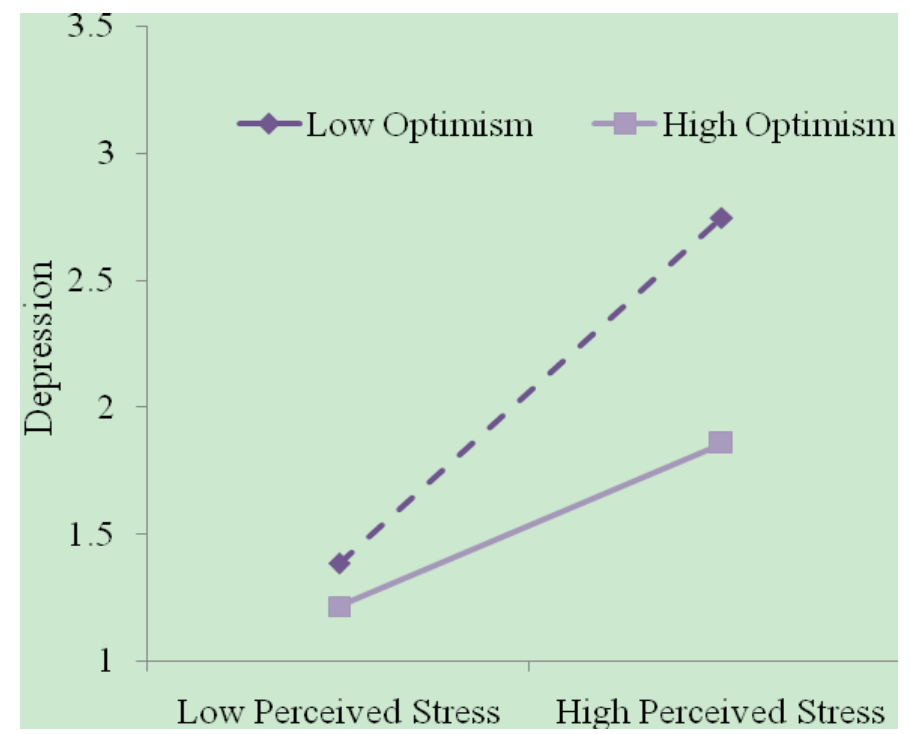

Figure 1. Interaction between participants' predicted values of self-reported depression and centered values on the perceived stress and optimism scales.

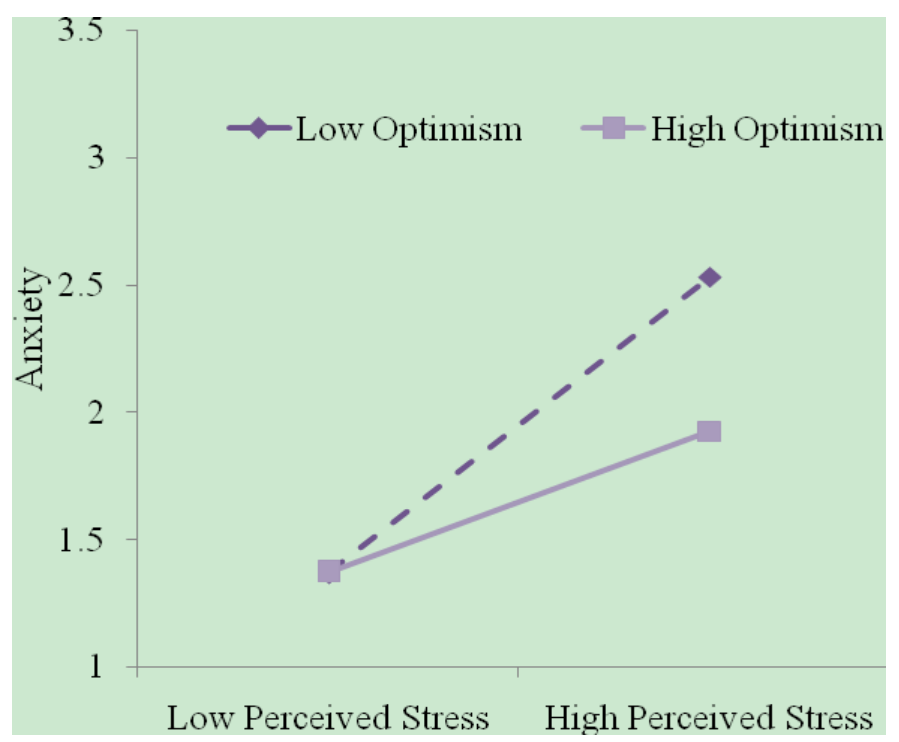

Figure 2. Interaction between participants' predicted values of self-reported anxiety and centered values on the perceived stress and optimism scales.

\section{Discussion}

The purpose of this study was to examine the main and interactive effects of perceived stress and optimism in predicting symptoms of depression and anxiety in college students. The results indicated that optimism has a direct effect on symptoms of depression and anxiety beyond what can be accounted for by perceived stress. Moreover, optimism moderates the relations between perceived stress and symptoms of depression and anxiety among participants in this study.

The results of the present study contribute to a converging body of evidence to support the role of optimism as a buffer of stress and psychological disorder (Chang, 1998; Chang \& Sanna, 2003). The pathways 
through which optimism influences adjustment to stress are in need of further study but the existing research suggests that optimism is related to students viewing their environment as less threatening and, consequently, being better adjusted psychologically than pessimists (Brissette et al., 2002; Chang, 1998; Chang \& Sanna, 2003). In other words, optimism may protect against symptoms of negative effect (e.g., depression, anxiety) by leading students to perceive their circumstances as less threatening.

The findings from this study suggest that it may be useful to consider interventions for students that focus on promoting optimism or to change pessimistic thoughts associated with negative feelings such as depressive symptoms. Interventions aimed at increasing an individual's positive view of the future may result in success for the treatment of students exhibiting symptoms of stress, depression, and anxiety. The ability to remain positive, to extract benefit from stressful life circumstances, and to have an adaptive, future-oriented perspective is an important factor in maintaining psychological health (Brissette et al., 2002; Hirsch, Wolford, LaLonde, Brunk, \& Parker Morris, 2007).

\section{References}

Aiken, L. S., \& West, S. G. (1991). Multiple regression: Testing and interpreting interactions. Thousand Oaks, C. A.: SAGE Publications.

ACHA-NCHA (American College Health Association-National College Health Assessment). (2010). Fall 2009 reference group data report. Baltimore: American College Health Association.

Baldwin, D. R., Chambliss, L. N., \& Towler, K. (2003). Optimism and stress: An African-American college student perspective. College Student Journal, 37, 276-286.

Brissette, I., Scheier, M. F., \& Carver, C. S. (2002). The role of optimism in social network development, coping, and psychological adjustment during a life transition. Journal of Personality and Social Psychology, 82, 102-111.

Chang, E. C. (1998). Does dispositional optimism moderate the relation between perceived stress and psychological well-being? Personality and Individual Differences, 25, 233-240.

Chang, E. C., \& Sanna, L. J. (2003). Experience of life hassles and psychological adjustment among adolescents: Does it make a difference if one is optimistic or pessimistic? Personality and Individual Differences, 34, 867-879.

Cohen, S., Kamarck, T., \& Mermelstein, R. (1983). A global measure of perceived stress. Journal of Health and Social Behavior, 24, 385-396.

Hirsch, J. K., Wolford, K., LaLonde, S. M., Brunk, L., \& Parker Morris, A. (2007). Dispositional optimism as a moderator of the relationship between negative life events and suicide ideation and attempts. Cognitive Therapy and Research, 31, 533-546.

Huan, V. S., Yeo, L. S., Ang, R. P., \& Chong, W. H. (2006). The influence of dispositional optimism and gender on adolescents' perception of academic stress. Adolescence, 41, 533-546.

Kassel, J. D., Bornovalova, M., \& Metha, N. (2006). Generalized expectancies for negative mood regulation predict change in anxiety and depression among college students. Behaviour Research and Therapy, 45, 939-950.

Lai, J. C. L. (2009). Dispositional optimism buffers the impact of daily hassles on mental health in Chinese adolescents. Personality and Individual Differences, 47, 247-249.

Lazarus, R. S., \& Folkman, S. (1984). Stress, appraisal, and coping. New York: Springer

Lovibond, P. F., \& Lovibond, S. H. (1995). The structure of negative emotional states: Comparison of the depression anxiety stress scales (DASS) with the beck depression and anxiety inventories. Behaviour Research and Therapy, 33, 335-343.

McCarthy, C. J., Fouladi, R. T., Juncker, B. D., \& Matheny, K. B. (2006). Psychological resources as stress buffers: Their relationship to university students' anxiety and depression. Journal of College Counseling, 9, 99-110.

Robotham, D., \& Julian, C. (2006). Stress and the higher education student: A critical review of the literature. Journal of Further and Higher Education, 30, 107-117.

Scheier, M. F., Carver, C. S., \& Bridges, M. W. (1994). Distinguishing optimism from neuroticism (and trait anxiety, self-mastery and self-esteem): A reevaluation of the life orientation test. Journal of Personality and Social Psychology, 67, 1063-1078.

Stewart-Brown, S., Patterson, J., Peterson, S., Doll, H., Balding, J., \& Regis, D. (2000). The health of students in institutes of higher education: An important and neglected public health problem? Journal of Public Health Medicine, 22, 492-299. 